
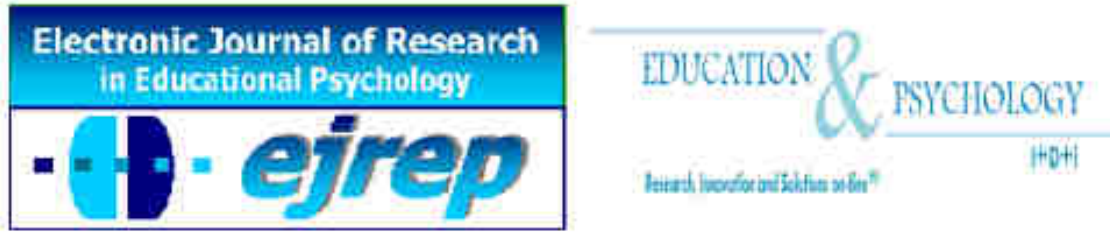

\title{
Evaluación del clima familiar creado por la gestión de los problemas de conducta, desde la perspectiva de los hijos
}

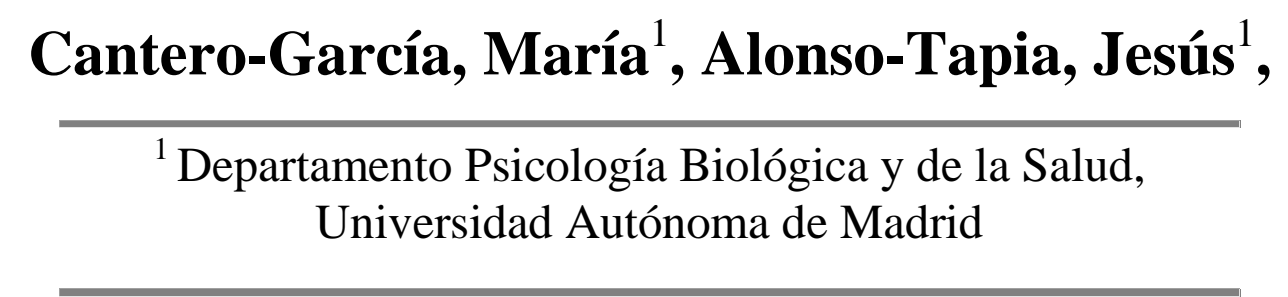

España

Correspondencia: María Cantero- García. Facultad de Psicología, Aula PDIF, C/ Ivan Pavlov, 6. Campus Cantoblanco. 28049 Madrid (España). E-mail: maria.cantero@uam.es

(C) Education \& Psychology I+D+i and Ilustre Colegio Oficial de la Psicología de Andalucía Oriental (Spain) 


\section{Resumen}

Introducción. Aunque se conocen las diferentes pautas de actuación que los padres suelen adoptar ante los problemas de conducta de los hijos, no se conoce si interactúan configurando lo que puede considerarse como clima familiar generado por la gestión de los problemas de conducta (CFGC).

Método. Para explorar este clima, en el presente estudio se ha desarrollado un cuestionario que recoge cuatro tipos de pautas de actuación: razonamiento vs castigo, estrategias debidas al estrés de los padres, reacción paciente, y estrategias constructivas de tipo positivo, estrategias cuya prevalencia e interacción definen el clima señalado, clima que se evalúa desde la perspectiva de los hijos sobre el mismo (CFGC-H). Un total 819 alumnos completaron el cuestionario. Se realizaron análisis factoriales confirmatorios, de validación cruzada, de fiabilidad y de rutas con variables latentes, utilizando la satisfacción de los hijos como criterio.

Resultados. Los resultados apoyan las hipótesis sobre la estructura del cuestionario y su validez predictiva, y sugieren la necesidad de actuar sobre los componentes del CFGC-H a la hora de diseñar intervenciones para mejorar el comportamiento de los hijos.

Discusión y conclusiones. Los resultados sugieren que la actitud positiva y la paciencia son factores importantes que contribuyen favorablemente a establecer un clima familiar positivo, en línea con lo encontrado en otras investigaciones. Por otro lado, todas aquellas estrategias de disciplina parental coercitivas como el castigo o las estrategias que contribuyen al aumento del estrés se asocian de forma negativa al clima familiar.

Palabras Clave: Clima familiar; estrategias de afrontamiento, problemas de conducta; estilos educativos; parentalidad positiva. 


\begin{abstract}
Introduction. Though different intervention patterns that parents use to employ for managing children behavior problems are known, it is not known whether they interact an how, configuring what can be considered as the family climate generated by the way in which children' behavior is managed" (FCBM).

Method. In this study, we developed a questionnaire that includes four kinds of patterns for coping with behavior problems: reasoning vs punishment, strategies due to parental stress, patience, and positive constructive strategies. The prevalence of and interaction among all these strategies are supposed to define the above-mentioned climate that, in this case, is assessed from the children's perspective (FCBM-C). A total of 819 students completed the questionnaire. Confirmatory factor analysis and cross validation analysis, reliability, and path analysis with latent variables using children's satisfaction as criterion were made.
\end{abstract}

Results. Results support the hypotheses about the structure of the questionnaire, and about its predictive validity, and suggest the need to act on the components of FCBM-C when designing interventions to improve children behavior.

Discussion and Conclusion. Results suggest that positive attitude and patience are the factors which most contribute to establish a positive family environment, in line with findings from other investigations. On the other hand, parental discipline strategies such as punishment or coercive strategies that contribute to increased stress are negatively realted to a positive family climate.

Keywords: Family climate; coping; behavioral problems; educational styles; positive parenting. 


\section{Introducción}

Los problemas de comportamiento de los hijos (rabietas, desobediencias, impulsividad o agresividad) constituyen una preocupación seria en el ámbito familiar (Cantero-García y Alonso-Tapia, 2017; Montiel-Nava, Montiel-Barbero y Peña, 2005; Robles y Romero Trianes, 2011) hasta el punto en que -de acuerdo con un estudio epidemiológico realizado con una muestra de 1220 padres y madres- el 52\% cree necesitar ayuda psicológica para resolver los problemas señalados (Cantero-García y Alonso-Tapia, 2016). El modo en que los padres se enfrentan a tales problemas -modo que configura uno de los aspectos de lo que se conoce como "clima familiar" - afecta no sólo al comportamiento de los hijos, sino su propio bienestar psicológico (Luengo Martín, 2014; Pérez, Menéndez y Hidalgo, 2014).

La importancia de los problemas de conducta ha llevado a la realización de numerosos estudios sobre la eficacia de distintas estrategias para el manejo de los mismos (WebsterStratton, Reid y Hammond, 2001; Romero, Villar, Luengo, Gómez-Fraguela y Robles, 2013), pero la investigación relativa al impacto que tiene el uso de dichas estrategias en el bienestar de los padres es escasa (Pérez-et al., 2014; Semke, Garbacz, Kwon, Sheridan y Woods, 2010; van Stejin, Oerlemans, van Aken, Buitelaar y Rommelse, 2013). Dado que tal impacto puede ser negativo, resulta necesario conocer no sólo la efectividad de los distintos modos de actuar frente a los problemas de conducta, sino también la repercusión que los mismos tienen sobre el estrés de los padres o, por el contrario, sobre su bienestar, al contribuir a la calidad del clima familiar.

\section{El clima familiar}

El clima familiar se define como el estado de bienestar positivo o negativo resultante del conjunto de patrones de interacción que se dan entre las personas de una familia tales como los tipos de comentarios sobre las calificaciones o el comportamiento, las ayudas, los límites, las sanciones, etc. (Alonso-Tapia, Simón y Asensio, 2013). Estas interacciones, dependiendo de su naturaleza, pueden tener efectos sobre múltiples variables tales como la motivación de los hijos por aprender (Alonso-Tapia, Simón y Asensio, 2013; Pomerantz, Grolnick y Pierce, 2005), los logros académicos (Jeynes, 2007), la autoeficacia, la implicación y la motivación intrínseca (Fan y Williams, 2010) o el comportamiento de los hijos (Leung, Tsang, Sin y Choi, 2015) 
En relación con los efectos sobre el comportamiento, estas interacciones pueden estar orientadas a facilitar el desarrollo y bienestar de la familia en la medida en que se traducen en actitudes de flexibilidad, cooperación, participación, interés, respeto y ayuda o, por el contrario, pueden interferir en el desarrollo del bienestar e influir de modo más negativo traduciéndose en comportamientos intolerantes, egocéntricos, disruptivos o violentos (Alonso-Tapia, 2012). Por este motivo, es necesario diferenciar entre clima familiar positivo y negativo.

El clima familiar positivo es el resultado de las prácticas de socialización de la familia que fomentan la confianza, la autonomía, la iniciativa y los lazos afectivos para el desarrollo de hijos e hijas estables y competentes. Algunas de estas prácticas son la escucha, el razonamiento, la sustitución del castigo por técnicas como el tiempo fuera (time out), el refuerzo de conductas positivas, el uso del modelado, los elogios, las reglas claras, etc. Hay evidencia empírica de que el clima familiar positivo es un factor protector frente a los problemas de comportamiento, $y$ ha mostrado ejercer una influencia significativa tanto en la conducta como en el desarrollo social, físico, afectivo e intelectual de los integrantes del núcleo familiar (Moreno, Estévez, Murgui y Musitu, 2009; Sanders, Kirby, Tellegen y Day, 2014). Las relaciones positivas entre padres/madres e hijos se han asociado con un mayor desarrollo de conductas prosociales en el ámbito familiar (Dolezal-Sams, Nordquist y Twardosz, 2009; Jaureguizar y Ibabe, 2012; Romano, Tremblay, Boulerice y Swisher, 2005).

Por el contrario, el clima familiar negativo es uno de los factores de riesgo más conocidos y se caracteriza por un elevado nivel de conflicto familiar y bajo nivel de cohesión. (Mitchell, Szczerepa, y Hauser-Cram, 2015). Algunas de las prácticas que forman el clima familiar negativo son la falta de reglas claras, un uso excesivo del castigo, excesiva autoridad, etc. Distintos estudios han mostrado que las prácticas parentales inadecuadas, tales como los castigos físicos, tienen consecuencias negativas a largo plazo en el ajuste psicológico de los hijos (Kazdín, 1997; Musitu y García, 2004; Gámez-Guadix, Straus, Carrobles, Muñoz-Rivas y Almendros, 2010; Algood, Harris y Hong, 2013). En consecuencia, si el clima familiar como ambiente de desarrollo es positivo, puede proporcionar a los niños factores protectores para su desarrollo, pero si es negativo puede actuar a su vez como fuente de estrés para los padres, repercutiendo no sólo en los padres sino también en los problemas de conducta de los hijos.

El clima familiar, sin embargo, no sólo afecta a los niños. Educar a un niño con problemas de comportamiento tales como frecuentes rabietas, desobediencias, impulsividad o 
agresividad ofrece numerosos retos a los padres, retos que, si no se saben afrontar, pueden tener repercusiones negativas en su equilibrio personal. Por ejemplo, los padres cuyos hijos presentan problemas de conducta suelen presentar tasas más altas de separación o divorcio y suelen estar más aislados, además de presentar niveles más altos de estrés (Montiel-Nava et al., 2005; Pérez-López, Rodríguez-Cano, Montealegre, Pérez-Lag, Perea y Botella, 2011). Estos hechos implican que la falta de estrategias de afrontamiento adecuadas puede incrementar los niveles de estrés parental, así como dificultar la solución de problemas de comportamiento que puedan presentar los hijos, estableciéndose un proceso autoretroalimentado en el que los problemas comportamentales de los hijos aumentan el estrés parental, que a su vez empeora tales problemas.

Por el contrario, si el reto que plantean los problemas de conducta se afronta adecuadamente, es posible que los padres lleguen a manifestar una notable resiliencia, la cual tiene efectos positivos sobre el bienestar personal (Prince-Embury y Saklofske, 2013). En consecuencia, resulta importante saber qué estrategias de manejo de los problemas de conducta y del estrés generado por estos son adecuadas y generan padres resilientes y qué estrategias resultan inadecuadas, dado que dependiendo de cuáles predominen en una familia dada, el clima familiar será más o menos positivo, y la resiliencia y el bienestar de los padres variarán de forma acorde.

Para poder investigar sobre el clima familiar generado por los modos de afrontar los problemas de conducta y su impacto sobre el estrés de los padres es necesario disponer de instrumentos de evaluación adecuados. El clima familiar generado por el manejo de los problemas de conducta puede evaluarse tanto a partir de la percepción de los padres como de la de los hijos. Por esta razón resulta imprescindible estudiar la percepción de ambos, dado que pueden no coincidir y que la validez de cada una de las perspectivas a la hora de decidir cómo actuar puede ser diferente. No existen en la actualidad instrumentos adecuados para realizar tal evaluación, y es nuestro objetivo desarrollar tal instrumento. El presente trabajo se centra en la percepción que los hijos tienen de las formas parentales de gestión del clima familiar. Ahora bien, ¿qué instrumentos existen que puedan servir de orientación para nuestro propósito y desde qué supuestos se han desarrollado?

\section{Instrumentos para la evaluación de los problemas de conducta}

Categorías de estilos parentales. Baumrind (1971), a partir de tres variables parentales básicas -control, comunicación e implicación afectiva-, identificó cuatro estilos parentales. 
Estos fueron: 1) autoritario -altos niveles de control, inflexibilidad (no razonamiento) y escaso afecto; 2) democrático -altos niveles de control, pero razonando mucho, y con mucho afecto; 3) permisivo -bajo control y mucho afecto y comunicación; y 4) negligente -bajo control, bajo razonamiento y bajo afecto. A su vez, estas características influyen en el bienestar psicológico de los padres y en su nivel de resiliencia.

Destacan también algunos de los instrumentos recogidos por Martín, Cabrera, León \& Rodrigo (2013):

Framework for the Assessment of Children in Need and their Families (FACNF). Desarrollado en Gran Bretaña (Department of Health et al. 2000), los autores de este instrumento proponen tres dominios para la evaluación familiar: 1) las necesidades evolutivas del menor, 2) las capacidades de los padres y 3) los factores ambientales y familiares. Con respecto a las competencias, el FACNF subraya la capacidad de los padres para ofrecer calidez emocional, estimular el desarrollo del menor, orientar y establecer límites y proporcionar un ambiente estable.

Comprehensive Family Assesment Guidelines for Child Welfare. Esta guía de evaluación desarrollada por el National Child Welfare Resource for Family-Centered Practice en Estados Unidos (Schene, 2005) permite evaluar la responsabilidad de los padres sobre las conductas de sus hijos y su motivación al cambio, la capacidad de conseguir que el niño vaya al colegio, la capacidad para controlar la expresión de ira, la capacidad de formar y mantener relaciones saludables y las habilidades de solución de problemas.

Standardized Assessment of Parenting, desarrollado por el New Zealand Children, Young Persons, and Their Families Services (Barber y Delfabbro, 2000). Este instrumento permite evaluar la capacidad parental para proporcionar cuidados básicos que satisfagan las necesidades infantiles a partir del análisis del funcionamiento del sistema familiar, las prácticas positivas y negativas de los padres y las técnicas de resolución de conflictos.

Escala de competencia y resiliencia parental para madres y padres en contextos de riesgo psicosocial (Martín et al., 2013). Desarrollada en Gran Canaria, esta escala permite evaluar las capacidades educativas, ligadas a los modelos de crianza y las de integración comunitaria, señaladas en el modelo de Barudy y Danthagnan (2010), así como aquellas competencias emotivas, cognitivas y conductuales que permiten construir el apego. También evalúa aquellas competencias que se consideran básicas desde el modelo británico (Department of Health et al., 2000), tales como la capacidad de asegurar las necesidades básicas del menor como la alimentación y la higiene, las competencias que permiten 
garantizar la seguridad y orientación del menor como el control y supervisión del comportamiento infantil y la organización de actividades de ocio con toda la familia.

Debido a que esta escala es de especial relevancia para el diseño de nuestro cuestionario, detallaremos algunos de los ítems de sus subescalas. En la escala de agencia parental (se siente capaz y eficaz como progenitor; percibe su rol parental de manera ajustada); promoción de la salud (muestra estrategias de afrontamiento ante situaciones de estrés, se preocupa por la higiene de los menores); organización doméstica (mantiene la casa limpia y ordenada, prepara comidas regularmente cada día); autonomía personal y búsqueda de apoyo (Tiene una visión positiva del niño y la familia, busca ayuda de personas significativas cuanto tiene problemas con sus hijos); habilidades educativas (controla y supervisa el comportamiento de sus hijos; muestra calidez y afecto en las relaciones con sus hijos; tiene expectativas de logro hacia sus hijos); desarrollo personal (reconoce y valora positivamente sus cualidades personales, muestra capacidad para resolver conflictos, se muestra flexible ante las dificultades); competencias cognitivas (es capaz de ponerse en el lugar del otro; es capar de autocorregirse cuando comete errores; es flexible para aplicar las pautas educativas en función de la edad, las características del niño y la situación); competencias emocionales (promueve relaciones de confianza y comunicación con sus hijos las; regula bien las emociones negativas); relación con la comunidad (se siente identificado e integrado en su comunidad; vive de manera estable en la comunidad) y por último, actitud hacia el servicio (tiene conciencia del problema; se muestra motivado para cambiar; tiene expectativas positivas hacia el futuro de los niños).

Como puede verse, la mayoría de las escalas que componen los instrumentos mencionados no son escalas de evaluación de la gestión de los problemas de conducta, sino del clima familiar en general. Las escalas relacionadas con la gestión de los problemas de conducta lo hacen de modo muy global, lo que no nos permite profundizar en las distintas pautas de actuación que se sabe que influyen en el clima familiar y en la gestión de los problemas de comportamiento. Además, proporcionan exclusivamente el punto de vista de los padres, que puede no reflejar realmente lo que ocurre, ya que la perspectiva de los hijos puede ser diferente, hecho del que ya hemos resaltado su importancia.

\section{Estructura del cuestionario propuesto}

A partir de las limitaciones de los cuestionarios existentes que se acaban de mencionar, se elaboró un cuestionario destinado a evaluar la percepción de los hijos sobre la 
gestión de los problemas de conducta que realizan los padres. Su estructura se recoge en la Figura 1, que muestra el modelo desarrollado cuya validación constituye el objetivo principal de este estudio. En este modelo, el "clima familiar de gestión de los problemas de conducta (CFGC) se define como el resultado de la interacción del grado en que los padres utilizan en conjunto los distintos tipos de estrategias mencionadas.

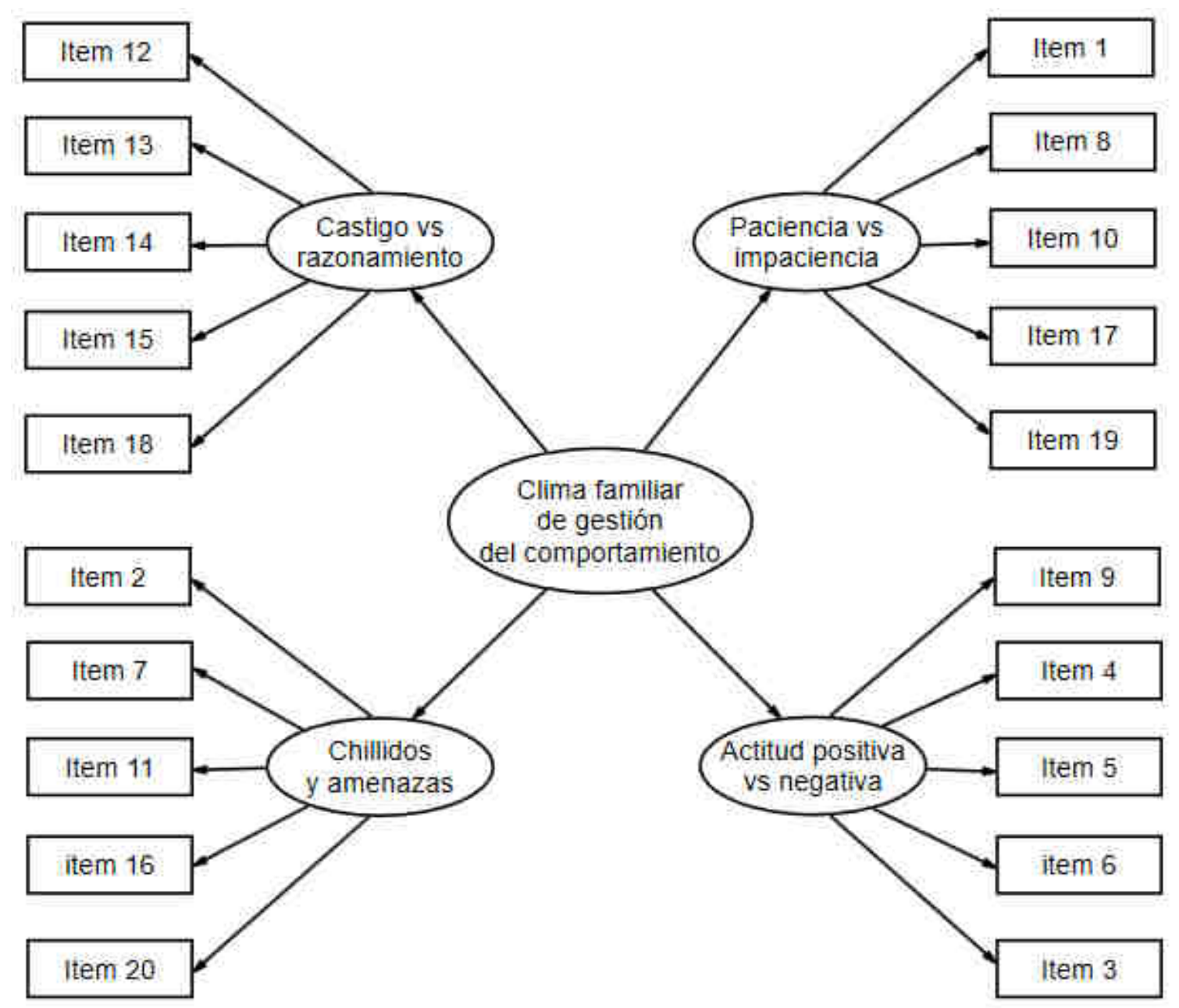

Figura 1. Modelo estructural del cuestionario de "Clima familiar de gestión del comportamiento" (CFGC)

Como puede apreciarse en la Figura 1 el cuestionario se compone de cuatro escalas que recogen los factores que contribuyen a explicar el clima familiar generado por la gestión de los problemas de conducta: Razonamiento vs castigo, Paciencia, Estrategias que contribuyen al aumento del estrés (chillidos, amenazas...) y estrategias que implican pensar en positivo -Actitud positiva-. La inclusión de las escalas de Paciencia, Actitud positiva, y Razonamiento vs castigo se inspira en el estudio de Parra y Oliva (2006), que mostró que los 
jóvenes que viven en un ambiente cálido y afectuoso tienen menos problemas conductuales, lo que ocurriría porque las buenas relaciones familiares aumentan el poder de influencia de los padres y madres sobre los hijos e hijas, disminuyendo la posible implicación en conductas antisociales. Las estrategias que contribuyen al aumento del estrés (chillidos, amenazas) constituyen también un factor clave a la hora de hablar del clima familiar, ya que no sólo contribuyen al empeoramiento de los problemas de conducta, sino que también aumentan el malestar psicológico de los padres.

Las escalas desarrolladas tienen por objetivo conocer las interacciones de los padres desde el punto de vista de los hijos, así como el grado en que dicho clima predice la satisfacción de éstos con el mismo.

\section{Objetivos e hipótesis}

Los objetivos, pues, de este estudio son: 1) conocer la percepción que tienen los hijos sobre la forma de actuar de sus padres frente a los problemas de conducta y 2) conocer los efectos de tales actuaciones en la satisfacción de los hijos. Con el fin de conseguir estos objetivos se han desarrollado dos pruebas (un cuestionario y una escala de satisfacción), y se han realizado los correspondientes análisis de validación que se exponen a continuación.

De acuerdo con el modelo recogido en la Figura 1 se espera que el clima familiar venga definido de forma positiva por las variables razonamiento vs castigo, paciencia, y actitud positiva, y de forma negativa por la reacción al estrés mediante chillidos y amenazas. Asimismo, se espera que cuanto más positiva sea la percepción del clima familiar, mayor será la satisfacción de los hijos.

\section{Método}

\section{Participantes}

Dos colegios concertados de Madrid y dos colegios públicos de Granada fueron elegidos por razones de conveniencia y proporcionaron el acceso a los estudiantes, en las etapas comprendidas entre $5^{\circ}$ de Primaria y $4^{\circ}$ de ESO. Participaron 407 chicos y 412 chicas $(\mathrm{N}=819)$, con edades comprendidas entre los 9 y 18 años (edad media de 13.17 años; $S D=$ 1.83), siendo la gran mayoría españoles (95.8\%). Los alumnos estaban distribuidos de la siguiente manera: 160 cursaban $5^{\circ}$ de primaria y $185,6^{\circ} ; 151$ se encontraban en $1^{\circ}$ de ESO, 120 en $2^{\circ}, 95$ en $3^{\circ}$ y 108 en $4^{\circ}$. 


\section{Instrumentos}

Cuestionario de clima familiar percibido por los hijos en relación a la gestión que realizan los padres ante los problemas de conducta (CFPC-H). Este cuestionario fue diseñado por los autores para el presente estudio. En primer lugar, se realizó la elaboración de los ítems en base a la bibliografía consultada. A continuación, se realizó un acuerdo interjueces en el que se escogieron los ítems que iban a formar parte del cuestionario. Posteriormente, el instrumento fue revisado por dos miembros del equipo de investigación. Incluye 20 ítems para evaluar la percepción de los hijos sobre las estrategias de manejo de los problemas de conducta utilizadas por los padres ante estos. Los ítems hacen referencia a cuatro estrategias: actitud positiva, razonamiento vs castigo, paciencia y estrés. El cuestionario incluye ítems formulados tanto de forma positiva como de manera negativa, que se responden señalando el grado de acuerdo con su contenido en una escala Likert de cinco puntos (siendo 1 totalmente en desacuerdo y 5 totalmente de acuerdo). Este cuestionario se incluye en el Apéndice A.

Cuestionario de satisfacción y cambio percibido por los hijos con la forma de gestión de los padres. Este instrumento, también diseñado por los autores, fue elaborado siguiendo el mismo procedimiento que el cuestionario anterior. Consta de seis ítems, tres formulados en sentido positivo y tres en sentido negativo, que evalúan el grado de satisfacción de los hijos con respecto a las actuaciones de sus padres. El formato de respuesta fue el mismo que en el cuestionario anterior. Este cuestionario se incluye en el Apéndice B.

\section{Procedimiento}

El estudio fue aprobado por el Comité de ética de la Universidad de los autores. Se contactó con 12 centros educativos, se les informó de los objetivos de la investigación y se pidió su colaboración. Cuatro centros aceptaron participar y remitieron a los padres y madres la carta de aceptación dónde se les invitaba a autorizar a su hijo a cumplimentar el cuestionario de forma anónima. Los niños y niñas autorizados cumplimentaron los cuestionarios en su aula de referencia durante una sesión de 30-40 minutos. Se indicó a los estudiantes qué debían responder pensando sobre todo en la persona -padre o madre- que más tiempo les dedicase, y que si no vivían con sus padres, debían rellenar el cuestionario 
pensando en las personas con las que vivían y que actuaban en la práctica como padres o tutores.

\section{Análisis de datos}

El grupo total se dividió aleatoriamente en dos submuestras $\left(n_{1}=409 ; n_{2}=410\right)$, una para el análisis inicial de la validez estructural del modelo y la otra para el análisis de validación cruzada. Para estudiar la validez estructural del modelo se realizó en primer lugar, el análisis factorial confirmatorio del modelo presentado en la Figura 1. Como método de estimación se utilizó máxima verosimilitud y se adoptaron los criterios descritos por Hair, Black, Babin and Anderson (2010) para aceptar o rechazar el modelo en base al ajuste del mismo $\left(\chi^{2} / g l<5\right.$; GFI, IFI y CFI > .90; RMSA <.08; SRMR <.08). Posteriormente, se realizó el análisis de validación cruzada, utilizando el mismo método de estimación y los mismos criterios de aceptación. En tercer lugar, se analizó la fiabilidad de cada una de las escalas de actuación frente a los problemas de conducta, así como la de la escala global. Finalmente, con el propósito de poner a prueba la validez predictiva del cuestionario, se realizaron dos análisis de rutas con variables latentes, con la satisfacción de los hijos como criterio y las diferentes estrategias de manejo de los problemas de conducta como predictores. El primer análisis sirvió para contrastar el modelo y, el segundo, para la validación cruzada.

\section{Resultados}

\section{Estructura factorial confirmatoria}

La Figura 2 muestra la estructura factorial del cuestionario y los índices de ajuste de los datos del análisis factorial confirmatorio realizado con la primera submuestra aleatoria $\left(n_{1}\right)$. El estadístico $\chi^{2}$ fue significativo $(p<.001)$, probablemente debido al tamaño de la muestra (Hair et al., 2010), pero la razón $\chi^{2} / g l$ y los índices GFI, RMSEA y SRMR están dentro de los límites de aceptación. Sin embargo, los índices IFI y CFI estuvieron ligeramente por debajo de dichos límites $\left(\chi^{2} / g l=2.69 ; \mathrm{GFI}=.90 ; \mathrm{IFI}=.86 ;\right.$ CFI: $.86 ;$ RMSEA $=.064 ;$ SRMR $=$ $.068)$. 


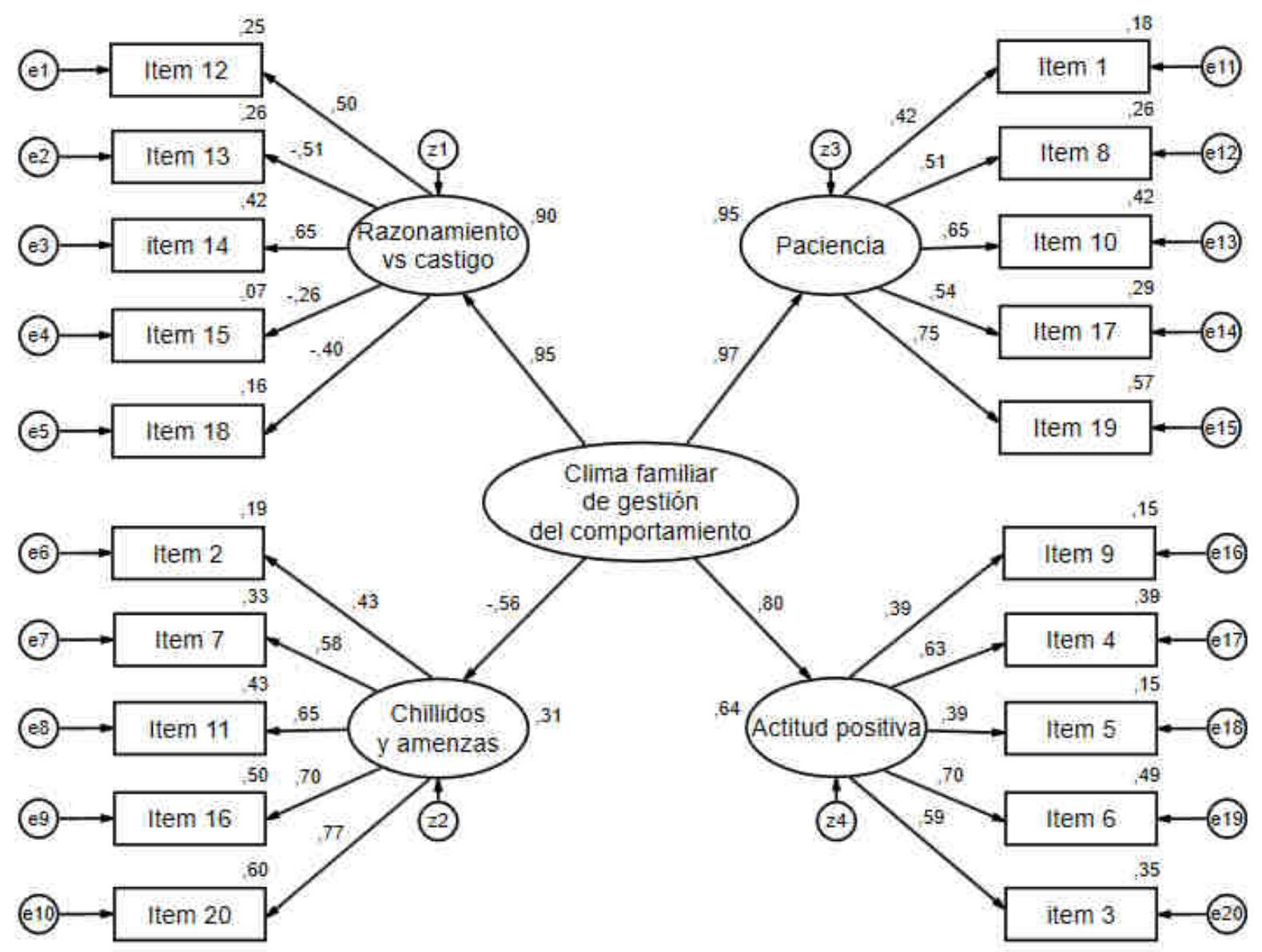

Figura 2. Cuestionario CFGC: Solución confirmatoria estandarizada correspondiente al modelo inicial

\section{Validación cruzada}

El análisis de validez cruzada fue realizado con las dos submuestras aleatorias $\left(n_{1}=\right.$ $\left.409 ; n_{2}=410\right)$. El estadístico $\chi^{2}$ fue significativo $(p<.001)$, probablemente debido al tamaño de la muestra, pero la razón $\chi^{2} / d f$, y los índices GFI, RMSEA y SRMR estuvieron dentro de los límites de aceptación del modelo. Los índices IFI y CFI estuvieron de nuevo por debajo de dichos limites $\left(\chi^{2} / g l=2.5\right.$; GFI $=.89$; IFI $\left.=.85 ; \mathrm{CFI}=.81 ; \mathrm{RMSEA}=.04 ; \mathrm{SRMR}=.07\right)$. Sin embargo, la comparación de los grupos puso de manifiesto que los índices de ajuste no decrecen significativamente al comparar modelos si se imponen restricciones para la aceptación de la igualdad de los resultados de las dos muestras en pesos de medida $\left(\Delta \chi^{2}=\right.$ $8,51, \mathrm{p}=.93)$, pesos estructurales $\left(\Delta \chi^{2}=10.05, p=.95\right)$, covarianzas estructurales $\left(\Delta \chi^{2}=\right.$ $11.74, p=.92)$, residuos estructurales $\left(\Delta \chi^{2}=12.23, p=.97\right)$ y residuos de medida $\left(\Delta \chi^{2}=\right.$ 
27.96, $p=.97$ ). Todo esto indica que no hay diferencias en el ajuste del modelo en ambas muestras, por lo que puede considerarse que está bien estimado.

\section{Fiabilidad}

La consistencia interna de la escala completa, evaluada mediante el índice $\alpha$ de Cronbach, fue de .84. Las escalas individuales obtuvieron los siguientes índices de fiabilidad: castigo vs razonamiento, $\alpha$ : .55; paciencia, $\alpha$ : .67; estrés, $\alpha$ : .77 y actitud positiva, $\alpha=.61$. En la escala criterio, el $\alpha$ de Cronbach fue de .74, lo que nos indica que es aceptable.

\section{Validez predictiva}

La Figura 3 muestra los resultados del análisis de rutas con variables latentes en que las dimensiones del clima familiar predicen la satisfacción de los hijos con el modo de actuar de los padres. En cuanto a los índices de ajuste, $\chi^{2}$ fue significativo $(p<.001)$, probablemente debido al tamaño de la muestra; $\chi^{2} / g l=2.9$, RMSEA $=.06$ y SRMR $=.07$, son adecuados, pero GFI $=.84$, IFI $=.82$ y CFI $=.82$ no alcanzaron por poco los límites estándar de aceptación, razón por la que se realizó el análisis de validación cruzada. No obstante, lo realmente importante es que el $98 \%$ de la varianza de la satisfacción de los hijos es explicada por las estrategias que estos perciben que los padres utilizan, siendo el razonamiento, la paciencia y la actitud positiva las que tienen un peso positivo y las estrategias que contribuyen al aumento del estrés (chillidos y amenazas) las que tienen un peso negativo. 


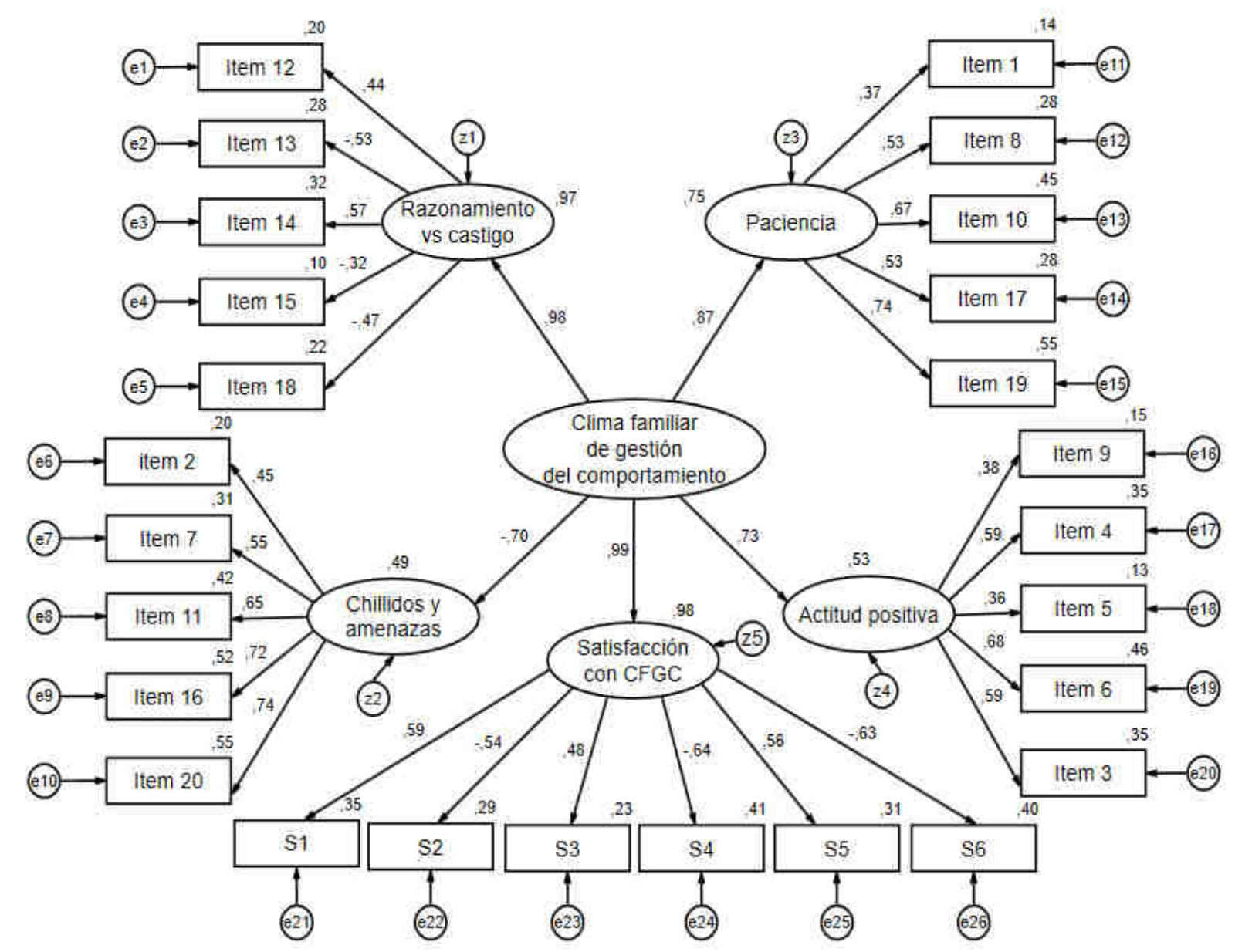

Figura 3. Resultado del análisis de rutas con variables latentes.

En el análisis de validez cruzada realizado con las dos submuestras aleatorias $\left(n_{1}=\right.$ 409; $\left.n_{2}=410\right)$, el estadístico $\chi^{2}$ fue significativo $(p<.001)$, probablemente debido al tamaño de la muestra (Hair et al., 2010), pero la razón $\chi^{2} / d f$, y los índices GFI, RMSEA y SRMR estuvieron dentro de los límites de aceptación del modelo. Los índices IFI y CFI estuvieron de nuevo por debajo de dichos limites $\left(\chi^{2} / g l=3.00 ; \mathrm{GFI}=.82 ; \mathrm{IFI}=.79 ; \mathrm{CFI}=.79 ;\right.$ RMSEA $=$ .05 ; SRMR $=.07)$. Sin embargo, la comparación de los grupos puso de manifiesto que los índices de ajuste no decrecen significativamente al comparar modelos si se imponen restricciones para la aceptación de la igualdad de los resultados de las dos muestras en los pesos de medida $\left(\Delta \chi^{2}=18.44, \mathrm{p}=.59\right)$, pesos estructurales $\left(\Delta \chi^{2}=22.26, p=.62\right)$, covarianzas estructurales $\left(\Delta \chi^{2}=24.25, p=.56\right)$, residuos estructurales $\left(\Delta \chi^{2}=24.52, p=.70\right)$ y residuos de medida $\left(\Delta \chi^{2}=52.11, p=.58\right)$. Todo esto indica que no existen diferencias en el ajuste del 
modelo en ambas muestras, por lo que puede considerarse que está bien estimado. Además, la varianza del criterio explicada por los predictores en ambos grupos fue la misma (98\%).

\section{Discusión y Conclusiones}

El objetivo de este estudio era desarrollar y validar un cuestionario para evaluar el clima familiar creado por el modo en que los padres manejan los problemas de conducta de los hijos, pero desde la perspectiva de los hijos, así como analizar el papel que dicho clima tiene en la satisfacción de éstos. Los resultados obtenidos indican que el cuestionario tiene una estructura factorial coherente, con índices estadísticos de ajuste aceptables, tiene una buena fiabilidad global y se relaciona del modo esperado con la satisfacción de los hijos. Las escalas incluidas, sin embargo, no deberían utilizarse de forma separada, ya que su consistencia interna no superó el valor de .70 salvo en el caso de las estrategias debidas al estrés.

Los resultados sugieren que la actitud positiva y la paciencia son factores importantes que contribuyen favorablemente a establecer un clima familiar positivo, en línea con lo encontrado en otras investigaciones, tales como las de Parra y Oliva (2006). El razonamiento, tal y como hemos comprobado en los estudios realizados por Torío et al. (2008), también se asocia con mejor competencia social, y predice la menor presencia de problemas de comportamiento. Por otro lado, todas aquellas estrategias de disciplina parental coercitivas como el castigo o las estrategias que contribuyen al aumento del estrés se asocian de forma negativa al clima familiar percibido por los hijos. (Algood, Harris y Hong 2013, GámezGuadix, Straus, Carrobles, Muñoz-Rivas y Almendros 2010; Kazdín, 1997; Musitu y García, 2004).

\section{Implicaciones}

Los resultados obtenidos tienen implicaciones teóricas, además de implicaciones para la evaluación y la intervención. Con respecto a las primeras, si bien se conocía el efecto aislado de algunas estrategias de gestión de los problemas de comportamiento (Torio et al. 2008), no había estudios que permitiesen evaluar si podía considerarse que contribuían a definir el clima familiar de gestión del comportamiento en su conjunto, dado que los estudios citados no daban una definición clara del mismo. Nuestros resultados sugieren que, al menos desde el punto de vista de los hijos, en la medida en que el uso de las distintas estrategias 
converge en una misma dirección puede tener un efecto diferente del que puede tener el uso de cada estrategia por separado. Esta implicación es válida tanto en relación con el comportamiento de los hijos -como sugiere el grado de satisfacción asociado a dicho clima-, como en relación con el equilibrio emocional de los padres, si bien los efectos aludidos no han sido comprobados, sino que se derivan de la naturaleza de las relaciones constatadas, por lo que deben ser investigados. Además, el clima definido por el modo de gestión del comportamiento es un componente del "clima familiar general" junto a otros, como por ejemplo, el "clima motivacional de la familia" (Alonso-Tapia, Simón y Asensio, 2013). Por consiguiente, para conocer el efecto del clima familiar general, conviene estudiar de qué modo interactúan sus distintos componentes.

En cuanto a las implicaciones para la evaluación, el hecho de disponer de instrumentos que consideren el punto de vista de los hijos respecto a las dinámicas familiares es positivo no sólo porque disponer de esta información permite predecir su nivel de satisfacción, sino porque tiene un valor diagnóstico respecto a las pautas de actuación paterna sobre los puede ser necesario actuar. El hecho de que esta información esté limitada a la perspectiva de los hijos sugiere la necesidad de obtener información de los propios padres sobre el clima familiar de gestión del comportamiento, objetivo para el que es necesario disponer de cuestionarios que en este momento no existen, tarea que queda por hacer.

Finalmente, en cuanto a las implicaciones para intervención, teniendo en cuenta la naturaleza de los componentes del clima de gestión del comportamiento identificados en este estudio, los programas dirigidos a las familias en las que existen problemas de conducta de los hijos, deberían, primero, fomentar estrategias y modos de actuación que fomente el uso del razonamiento y la evitación de medidas de tipo punitivo; segundo, facilitar el desarrollo de comportamientos asertivos que sustituyan a las estrategias debidas al estrés, basadas en la expresión emocional, y tercero, fomentar el desarrollo de la paciencia y la actitud positiva necesarias para gestionar los problemas de comportamiento de modo que las repercusiones sean positivas, como también sugieren algunos estudios recientes (Rodrigo, 2015; Rodrigo, Máiquez, Martín y Byrne, 2008) 


\section{Limitaciones y prospectivas}

Este estudio cuenta con ciertas limitaciones que merecen atención. En primer lugar, el muestreo por conveniencia de los colegios y su decisión sobre participar ha podido sesgar los resultados obtenidos y por tanto limitar la generalizabilidad de estos. Asimismo, la decisión de los padres sobre la participación de sus hijos ha podido resultar en que solo aquellos alumnos con padres altamente motivados hayan participado en el estudio. Una tercera limitación tiene que ver con no haber analizado por separado las estrategias del padre y la madre. La cuarta limitación tiene que ver con la baja fiabilidad de algunas de las escalas específicas, hecho que podría afectar al resto de resultados obtenidos. Estudios futuros deberían tener en cuenta estas limitaciones. Concretamente, debería aumentarse la fiabilidad de las escalas de actitud positiva, paciencia y razonamiento/castigo añadiendo ítems de contenido apropiado. Además, este estudio no ha medido específicamente la resiliencia de los padres, por lo que resulta necesario hacerlo en futuros estudios para poder conocer qué relación hay entre las estrategias de manejo de los problemas y la resiliencia. Esto permitirá, en última instancia, desarrollar intervenciones eficaces que contribuyan tanto al bienestar psicológico de los padres como al clima familiar positivo.

\section{Referencias}

Algood, C. L., Harris, C., y Hong, J.S. (2013). Parenting success and challenges for families of children with disabilities: An ecological system analysis. Journal of Human Behavior in the social environment, 23(2), 126-136. Doi: 10.1080/10911359.2012.747408.

Alonso-Tapia, J. (2012). Evaluación psicopedagógica y orientación educativa. Madrid: Síntesis.

Alonso-Tapia, J., Nieto, C. y Ruiz, M. A. (2013). Measuring Subjective Resilience despite Adversity due to Family, Peers and Teachers. Spanish Journal of Psychology, 16, 1-13.

Alonso-Tapia, J., Simón, C. y Asensio, C. (2013). Development and Validation of the Family Motivational Climate Questionnaire (FMC-Q). Psicothema, 25(2), 266-274. Doi: 10.7334/psicothema2012.18.

Barber, J. G. y Delfabbro, P. H. (2000). The assessment of parenting in child protection cases. Research in Social Work Practice, 10, 243-256.

Barudy, J. y Danthagnan, M. (2010). Los desafíos invisibles de ser madre o padre. Manual de evaluación de las competencias y la resiliencia parental. Barcelona: Gedisa editorial. 
Baumrind, D. (1971). Current patterns of parental authority. Developmental Psychology Monographs, 4(1), 1-103.

Cantero-García, M. y Alonso-Tapia, J. (2017). Cuestionario breve de respuesta parental ante el comportamiento disruptivo (RPCD): Perspectiva de los padres. Anales de psicología, 33(3), 689-696. Doi/10.6018/analesps.33.3.266971.

Cantero-García, M. y Alonso-Tapia, J. (En revisión). Estudio epidemiológico sobre los problemas de comportamiento en la infancia y en la adolescencia.

Department of Health, Department for Education and Employment, and Home Office (2000). Framework for the Assessment of Children in Need and their Families. London: The Stationery Office.

Dolezal-Sams, J. M., Nordquist, V. M. y Twardosz, S. (2009). Home environment and family resources to support literacy interaction: examples from families of children with disabilities. Early Education and Development, 20(4), 603-630.

Fan, W. y Williams, C. M. (2010). The effects of parental involvement on students 'academic self-efficacy, engagement and intrinsic motivation. Educational Psychology, 30(1), 53-74. Doi: 10.080/10409280802356661

Gámez-Guadix, M., Strauss, M. A., Carrobles, J. A., Muñoz-Rivas, M. y Almendros, C. (2010). Corporal punishment and long-term behavior problems: The moderating role of positive parenting and psychological aggression. Psicothema, 22, 529-536.

Hair, J. F., Black, W. C., Babin, B. J. y Anderson, R. E. (2010). Multivariate data analysis. Upper Saddle River, NJ: Pearson-Prentice Hall.

Jaureguizar, J. y Ibabe, I. (2012). Conductas violentas de los adolescentes hacia las figuras de autoridad: el papel mediador de las conductas antisociales. Revista de Psicología Social, 27, 7-24. Doi: 10.1174/021347412798844088

Jeynes, W. H. (2007). The relationship between parental involvement and urban secondary school student academic achievement. A meta-analysis. Urban Education, 42(1), 82-110.

Kazdín, A. E. (1997). Practitioner review: Psychosocial treatments for conduct disorder in children. Child Psychology and Psychiatry, 38(2), 161 -178.

Leung, C., Tsang, S., Sin, T. C. S. y Choi, S. (2015). The efficacy of parent-child interaction therapy with Chinese families: Randomized controlled trial. Research on Social Work Practice, 25(1), 117-128. Doi: 10.1177/1049731513519827

Luengo, M. A. (2014). Cómo intervenir en los problemas de conducta infantiles. Padres y Maestros, 356, 37-43. Doi: 10.14422/pym.v0i356.3071 
Martín, J. C., Cabrera, E., León., J. y Rodrigo, M. J. (2013). La escala de competencia y resiliencia parental para madres y padres en contextos de riesgo psicosocial. Anales de Psicología, 29(3), 886-896. Doi: 10.6018/analesps.29.3.150981

Mitchell, D., Szczerepa, A y Hauser-Cram, P. (2015). Spilling over: Partner parenting stress as a predictor of family cohesion in parents of adolescents with developmental disabilities. Research in Developmental Disabilities, 49-50, 258-267.

Montiel-Nava, C., Montiel-Barbero, I. y Peña, A. (2005). Clima familiar en el trastorno por déficit de atención-hiperactividad. Psicología Conductual, 13(2), 297-310.

Moreno, D., Estévez, E., Murgui, S. y Musitu, G. (2009). Relación entre el clima familiar y el clima escolar: el rol de la empatía, la actitud hacia la autoridad y la conducta violenta de la adolescencia. International Journal of Psychological Therapy, 9(1), 123-136.

Musitu, G. y García, F. (2004). Consecuencias de la socialización familiar en la cultura española. Psicothema, 16, 288-293.

Parra, A. y Oliva, A. (2006). Un análisis longitudinal sobre las dimensiones relevantes del estilo parental durante la adolescencia. Infancia y Aprendizaje, 29, 453-470. Doi: $10.1174 / 021037006778849594$

Pérez, J., Menéndez, S. e Hidalgo, M. V. (2014). Estrés parental, estrategias de afrontamiento y evaluación del riesgo en madres de familias en riesgo usuarias de los servicios sociales. Psychosocial Intervention, 23, 25-32.

Pérez-López, J., Rodríguez-Cano, R. A., Montealegre, M. P., Pérez-Lago, M., Perea, L. P. y Botella, L. (2011). Estrés adulto y problemas conductuales infantiles percibidos por sus progenitores. International Journal of Developmental and Educational Psychology, 1(1), 531-540.

Pomerantz, E. M., Grolnick, W. S. y Price, C. E. (2005). The role of parents in how children approach achievement. En A. J. Elliot y C.S Dweck (Eds.), Handbook of competence and motivation (pp. 259-296). New York, NY: Guilford Press.

Prince-Embury, S., y Saklofske D.H. (Eds.) (2013). Resilience in children, adolescent and adults: Translating research into practice. New York, NY: Springer.

Robles, Z. y Romero-Trianes, E. (2011). Programas de entrenamiento para padres de niños con problemas de conducta: una revisión de su eficacia. Anales de psicología, 27(1), 86101.

Rodrigo M. J., Máiquez, M. L., Martín, J. C. y Byrne, S. (2008). Preservación Familiar. Un enfoque positivo para la intervención con familias. Madrid: Pirámide. 
Rodrigo, M. J. (2015). Manual práctico de parentalidad positiva. Madrid: Síntesis.

Romano, E., Tremblay, R., Boulerice, B. y Swisher, R. (2005). Multilevel Correlates of Childhood Physical Aggression and Prosocial Behavior. Journal of abnormal child psychology, 33(5), 565-578. Doi: 10.1007/s10802-005-6738-3

Romero, E., Villar, P., Luengo, M. A., Gómez-Fraguela, J. A. y Robles, Z. (2013). EmPeCemos. Programa para la intervención en los problemas de conducta infantiles. Madrid: Tea Ediciones.

Sanders, M. R., Kirby, J. N., Tellegen, C. L. y Day, J. J. (2014). The Triple P-Positive Parenting Program: A systematic review and meta-analysis of a multi-level system of parenting support. Clinical Psychology Review, 34, 337-357.

Schene, P. (2005). Comprehensive Family Assessment Guidelines for Child Welfare. National Child Welfare Resource Center for Family-Centered Practice. Recuperado de: http://www.acf.hh.gov/programs/cb/pubs/family_assessment/

Semke, C. A., Garbacz, S. A., Kwon, K., Sheridan, S. M. y Woods, K. E. (2010). Family involvement for children with disruptive behaviors: The role of parenting stress and motivational beliefs. Journal of School Psychology, 48, 293-312.

Torío, S., Peña, J. C., Caro, M. (2008). Estilos de educación familiar. Psicothema, 20(1), 6270.

Van Stejin, D., Oerlemans, A., van Aken, M., Buitelaar, J. y Rommelse, N. (2014). The reciprocal relationship of $\mathrm{ASD}$, ADHD, depressive symptoms and stress in parents of children with ASD and/or ADHA. Journal of Autism Developmental Disorder, 44, 10641076. Doi: 10.1007/s10803-013-1958-9

Webster-Stratton, C., Reid, M. J., y Hammond, M. (2001). Preventing conduct problems, promoting social competence: A parent and teacher training partnership in Head Start. Journal of Clinical Child Psychology, 30, 283-302. 
Apéndice A

\section{CUESTIONARIO DE CLIMA FAMILIAR DE MANEJO DE PROBLEMAS DE CONDUCTA}

\section{(Para niños de 11 a 16 años)}

\section{Instrucciones}

A continuación encontrarás una serie de afirmaciones que tienen que ver con modos de actuar y reaccionar de los padres frente a los problemas que a veces plantea nuestro modo de comportarnos. Conocerlo ayudará a saber cómo ayudarles y cómo ayudar a los hijos a convivir mejor. En relación con cada afirmación, señala el grado en que estés de acuerdo utilizando la siguiente escala.

A

$\mathrm{B}$

C

D

$\mathrm{E}$

Totalmente en desacuerdo Bastante en desacuerdo Indiferente Bastante de acuerdo Totalmente de acuerdo

1. Cuando me porto mal mis padres intentan hacerme pensar porque me he portado así.

2. Mi comportamiento suele poner nerviosos a mis padres

3. Aunque no me porte todo lo bien que debería mis padres siguen con ganas de hacer actividades conmigo.

4. A pesar de que me porte mal mis padres me siguen brindando su apoyo y afecto

5. Mis padres no se desaniman cuando no me porto como ellos esperan.

6. Aunque a veces me porte mal y mis padres se enfaden les encanta realizar actividades conmigo

7. Cuando no atiendo las órdenes de mis padres ellos gritan y se enfadan.

8. Cuando me porto bien mis padres me demuestran lo felices que se sienten.

9. Cuando me rebelo contra mis padres (contesto, grito, no les obedezco), ellos actúan con calma y firmeza.

10. Cuando me muestro muy inquieto o nervioso mis padres saben cómo relajarme.

11. Cuando estoy muy enfadado mis padres pierden los nervios.

12. Mi padre/madre con frecuencia suelen hacerme pensar sobre las cosas que he hecho mal.

13. Mi padre/madre siempre están enfadados y por eso me castigan.

14. Cuando hay algún problema mi padre/madre suelen sentarse a hablar conmigo de manera tranquila.

15. Mi padre/madre no me llevan a hacer la compra, o con sus amigos por mi mala actitud o desobediencia.

16. Cuando mi padre/madre se enfada conmigo suele chillar, gritarme o insultarme.

17. Mi padre/madre siempre están dispuestos a darme cariño aunque a veces los desespero cuando desobedezco.

18. Mis padres discuten con frecuencia a causa de mi mal comportamiento o rebeldía.

19. A pesar de que me porte mal mi padre/madre actúa con paciencia y saben animarme.

20. Ante mis rebeldías o desobediencias mi padre/madre se pone muy nervioso.

\section{Apéndice B}

\section{CUESTIONARIO DE SATISFACCIÓN}

1. Gracias al interés y tiempo que mis padres me dedican, incluso si me porto mal, cada vez confío más en ellos

2. Como mis padres suelen enfadarse conmigo cuando hago algo mal, cada vez nos llevamos peor.

3. Gracias a que, si les doy problemas, mis padres me hablan sin gritarme, razonando, intento dar lo mejor de mí

4. Como mis padres no saben qué hacer cuando les doy problemas, cada vez paso más de ellos.

5. Gracias a que cuando me porto mal mis padres actúan con serenidad y firmeza, cada vez me porto mejor.

6. Cuando hago algo mal, mis padres suelen perder los nervios, y eso hace que me porte peor. 